dust. Pathologists never report the presence of a possible metal-related aetiology in a specimen by lack of available technology. Therefore, several respiratory diseases are considered idiopathic. We recently developed an all-optical method, fully compatible with standard microscopy systems, for multi-elemental imaging of biological tissues.

Methods Our instrument is based on Laser Induced Breakdown Spectroscopy (LIBS) and allows the in situ imaging and quantification of the elements of the periodic table within biological tissues. A laser is focused on the sample surface. Elemental images (maps) are obtained by scanning the surface of the specimen. Spectrometers collect the signal of various elements such as Fe-Ca-Na-P-Mg$\mathrm{Zn}-\mathrm{Al}-\mathrm{Mn}-\mathrm{Co}-\mathrm{Si}-\mathrm{Cr}-\mathrm{Ti}$ and $\mathrm{Cu}$ in the tissue.

Results We identified and mapped the presence of high levels of $\mathrm{Si}$ and $\mathrm{Al}$, but also $\mathrm{Ti}$, or $\mathrm{Cr}$ in lung and mediastinal lymph nodes biopsies from exposed workers suffering from sarcoidosis or idiopathic pulmonary fibrosis. We found different elements including Lithium in the lymph node of a sarcoidosis patient working in a lithium-battery factory. We were also able to image the presence of Beryllium in the lungs of a patient with Chronic Beryllium Disease. As a matter of fact, Lithium and Beryllium are light-weight elements not detectable by any other elemental imaging technique. We will describe a panel of recent results obtained with LIBS, and confirmed with electron microscopy, focusing on respiratory diseases.

Discussion This laser spectrometry technique is versatile because almost any element can be imaged with high sensitivity. These results demonstrate the strong potential of this disruptive technology as a complementary tool for routine classical in-situ pathology examination of human tissues embedded in paraffin, especially for idiopathic respiratory diseases related to occupational or environmental exposure to metals, dust, or particles.

\section{DIFFERENTIAL ASSOCIATION OF LUNG FUNCTION IMPAIRMENT AND RISK COMMUNICATION ON MENTAL HEALTH}

Lang Jessica*, Felten Michael, Kraus Thomas. Institute of Occupational Medicine, RWTH Aachen University, Aachen, Germany

\subsection{6/oemed-2018-ICOHabstracts. 1251}

Introduction The knowledge of previous exposure to a hazardous material like asbestos may lead to chronic psychological strain (Lebovits, Byrne, \& Strain, 1986). Specifically, the information about an increased cancer risk can trigger emotional responses such as symptoms of depression and anxiety (Dilling, Mombour, \& Schmidt, 1993) which in turn activate coping mechanisms. This applies in particular to those individuals who a have developed non-malignant changes like lung fibrosis and pleural plaques (Gietmann, Gerd Meier, \& Trotschler, 1993). Resulting lung function impairment due to asbestos related diseases might occur even years after cessation of exposure. Consequently, it cannot be excluded that poor psychological well-being may also be associated with obstructive or restrictive lung function impairment.

Methods The 619 male study participants (M_age $=66.3$ years, $\mathrm{SD}=9.6$ ) attended a screening program for asbestos related diseases. Routine examinations included lung function testing and validated questionnaires for mental health (depression and anxiety) and coping. Hierarchical regression analyses were conducted.

Results The strongest predictor for mental health was the functional impairment due to restriction (e.g. for depression: beta $=0.21, t=3.15$ ). For predicting coping behaviour, the presence of a non-malignant asbestos related disease was most significant (e.g., for avoidant coping: beta=0.29, $\mathrm{t}=4.11$ ).

Discussion The presence of psychological health symptoms (i. e., depression, anxiety) is associated with ventilation problems, whereas the knowledge of an already initiated tissue change resulting from the asbestos exposure - is primarily associated with mental strain. Specifically, the affected individuals are more prone to intrusive thoughts and engage more in coping behaviours. As an implication, physicians should be sensitised about possible consequences of risk communication and functional restrictions in order to counteract excessive fear or anxiety.

\section{DISTRIBUTION OF SENSITIZER-INDUCED OCCUPATIONAL ASTHMA IN R. MACEDONIA IN THE PERIOD 2005-2016 BY OCCUPATION}

Jordan Minov*, Jovanka Karadzinska-Bislimovska, Sasho Stoleski, Dragan Mijakoski, Aneta Atanasovska. Institute for Occupational Health of RM - Skopje, Skopje, Macedonia

\subsection{6/oemed-2018-ICOHabstracts. 1252}

Introduction Occupational asthma (OA) became an important public health problem worldwide in the last few decades. From two different OA types, sensitizer-induced OA accounts for approximately $90 \%$ of all OA cases.

Objective To present the distribution of sensitizer-induced OA by occupation in R. Macedonia in the period 2005-2016.

Methods Sensitizer-induced OA was diagnosed by serial measurements of peak expiratory flow rate (PEFR) at and away from work or by combination of serial PEFR measurements at and away from work and non-specific bronchial provocation at and away from work in subjects with diagnosed asthma and work-relatedness of the symptoms.

Results The annual incidence rate of the diagnosed sensitizerinduced $\mathrm{OA}$ in the mentioned period varied from 1.8/100,000 working population in 2013 to $2.8 / 100,000$ in 2006. Sensitizer-induced OA in bakers, cleaners, textile workers and agricultural workers accounted up to more than a third of the all diagnosed cases. Atopy was registered in approximately a half of the sensitized-induced OA cases. Majority of the cases with sensitizer-induced $\mathrm{OA}$ caused by high-molecular-weight (HMW) agents (i.e. OA in bakers, textile workers, tanners, herbal and fruit tea processors, and health care workers) was atopics and had positive prick tests to occupational allergens.

Conclusion Our findings indicate the sectors with highest occurrence of sensitizer-induced $\mathrm{OA}$ in $\mathrm{R}$. Macedonia in the period 2005-2016. The data obtained enable directing of adequate activities to prevent developing of the disease, as well as to identify affected workers and to prevent further respiratory impairment. 